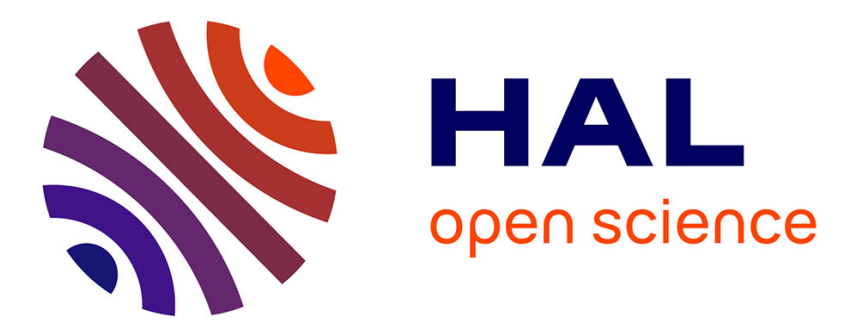

\title{
True Pareto Fronts for Multi-Objective AI Planning Instances
}

Alexandre Quemy, Marc Schoenauer

\section{To cite this version:}

Alexandre Quemy, Marc Schoenauer. True Pareto Fronts for Multi-Objective AI Planning Instances. European Conference on Combinatorial Optimization - EvoCOP, Apr 2015, Copenhague, Denmark. pp.197-208. hal-01109777

\section{HAL Id: hal-01109777 https://hal.science/hal-01109777}

Submitted on 27 Jan 2015

HAL is a multi-disciplinary open access archive for the deposit and dissemination of scientific research documents, whether they are published or not. The documents may come from teaching and research institutions in France or abroad, or from public or private research centers.
L'archive ouverte pluridisciplinaire HAL, est destinée au dépôt et à la diffusion de documents scientifiques de niveau recherche, publiés ou non, émanant des établissements d'enseignement et de recherche français ou étrangers, des laboratoires publics ou privés. 


\title{
True Pareto Fronts for Multi-Objective AI Planning Instances
}

\author{
Alexandre Quemy and Marc Schoenauer \\ TAO Project, INRIA Saclay \& LRI - Univ. Paris-Sud and CNRS, Orsay, France \\ \{alexandre.quemy, marc.schoenauer\}@inria.fr
}

\begin{abstract}
Multi-objective AI planning suffers from a lack of benchmarks with known Pareto Fronts. A tunable benchmark generator is proposed, together with a specific solver that provably computes the true Pareto Front of the resulting instances. A wide range of Pareto Front shapes of various difficulty can be obtained by varying the parameters of the generator. The experimental performances of an actual implementation of the exact solver are demonstrated, and some large instances with remarkable Pareto Front shapes are proposed, that will hopefully become standard benchmarks of the AI planning domain.
\end{abstract}

\section{Introduction}

Contrary to single objective problems, Multi-Objective Problems (MOP) involve several contradictory criteria to be optimized. This distinction entails a modification of the concept of optimality itself: the optimal solution of a MOP is not a single solution but a set of solutions that represents trade-offs known as the Pareto Set. This set is made of the non-dominated points of the search space, i.e. the solutions that cannot be improved w.r.t. one objective without deteriorate at least another one. Formally, $x$ dominates $y$ if $\forall i \in\{1, \ldots, n\}, f_{i}(x) \succeq f_{i}(y)$ and $\exists j \in\{1, \ldots, n\}, f_{j}(x) \succ f_{j}(y)$. The projection of the Pareto Set over the objective space is called the Pareto Front.

Many benchmark suites exist for continuous multi-objective optimization (the famous ZDT [9], IHR [1], ...), for which the exact Pareto Front can be analytically computed, and with known difficulties (e.g. dimensionality, shape of the Pareto Fronts, existence of local Pareto-optima, ... ). For combinatorial optimization, however, the situation is not yet so clear, and whereas there exist famous benchmark problems of all sizes, their true Pareto Fronts are only exactly known for the simplest problems (see e.g., MOCOLIB ${ }^{1}$, offering several instances of several well-known combinatorial benchmark problems).

The benchmark suite introduced in the present work is concerned with $A I$ planning: A planning domain $D$ is defined by a set of predicates that define the state of the system when instantiated and a set of possible actions that can be triggered in states where their pre-conditions are satisfied, resulting in a new state. A planning problem instance $\mathcal{P}_{D}(I, G)$ is defined on a given planning domain $D$ by a list of objects, used to instantiate the predicates to define the

\footnotetext{
${ }^{1}$ http://www .mcdmsociety.org/MCDMlib.html
} 
states, an initial state $I$ and a goal state $G$. The aim is to come up with an optimal feasible plan, i.e., a set of actions that, when applied in turn to the initial state, lead the system to the goal state, and is optimal w.r.t. a given measure: the number of actions, or the total cost of the plan when actions have nonuniform costs, or the total makespan (total duration of the plan) when actions have durations, and can be run in parallel.

MiniZenoTRAVEL is a simple temporal planning domain related to logistics, inspired by the well-known ZENOTRAVEL problem introduced in the 3rd edition of the IPC series ${ }^{2}$. It involves cities, passengers, and planes (see e.g., Figure 1); Planes can fly from one city to another when a link exists (on Figure 1, the flight duration is attached to the link); Planes fly either empty, or carrying a unique passenger - and these are the only possible actions. A MiniZenoTravel instance is defined by the number of cities and the graph of the possible flights between them, a number of passengers and a number of planes. In the initial state $I$, all passengers and planes are in city $c_{I}$, and in the goal state $G$, all passengers must be in city $c_{G}$. Previous work proposed a multi-objective version of these benchmarks called MultiZenoTravel, by adding a cost for landing in some cities: the second objective is to minimize the total cost of the plan $[8,2]$. The latter work demonstrated that such problems could provide Pareto Fronts of various shapes and difficulties. However, the authors were only able to provide the exact Pareto Front for very small instances, due to the combinatorial explosion of the solution space.

The present work formally analyzes the MultiZenoTravel benchmarks and provides an algorithm to compute their true Pareto fronts in reasonable time, even for very large instances. Beyond providing a generic way to generate Pareto Fronts of tunable complexities for AI Planning, the proposed MULTIZENOTRAVEL benchmarks will allow testing different multi-objective optimization algorithms, from generic decomposition methods (weighted sum aggregation, Tchebycheff decomposition, Boundary Intersection approach - see e.g., [6]) to Pareto-based Evolutionary Algorithms, on complex benchmarks for which the Pareto Front is exactly known.

The paper is organized as follows: Section 2 formally presents the MuLTIZENoTRAVEL benchmark, proving some properties of their Pareto optimal plans. Building on these properties, Section 3 proposes the ZENOSOLVER algorithm to actually derive the true Pareto Front for these instances. Sample experimental results demonstrate the diversity of Pareto Fronts that can be obtained, and gives performance measurements of its complexity on large instances.

\section{MultiZenotravel problem}

\subsection{Instances}

Let us introduce some notations related to the planning problem briefly presented in the introduction: a MultiZenoTravel instance (Figure 1) is defined by the following elements:

\footnotetext{
${ }^{2}$ http://ipc.icaps-conference.org/
} 


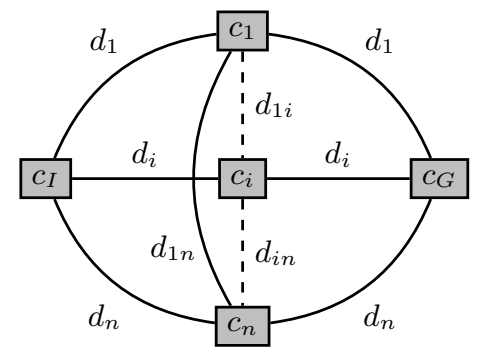

Fig. 1: A schematic view of a general MultiZenoTravel problem.

- $n$ central cities, organized as a clique in which every node is connected to $C_{I}$ and $C_{G}$, respectively the initial city and the goal city.

$-c \in\left(\mathbb{R}^{+}\right)^{n}$, where $c_{i}$ is the cost for landing in $C_{i}$.

- $D \in\left(\mathbb{R}^{+}\right)^{n \times n}$, where $D_{i j}$ is the flying time between $C_{i}$ and $C_{j}$.

- $d^{I} \in\left(\mathbb{R}^{+}\right)^{n}$, where $d_{i}^{I}$ is the flying time between $C_{I}$ and $C_{i}$.

$-d^{G} \in\left(\mathbb{R}^{+}\right)^{n}$, where $d_{i}^{G}$ is the flying time between $C_{i}$ and $C_{G}$.

- $p$ planes, initially in $C_{I}$, that have a capacity of an unique person.

$-t$ persons, initially in $C_{I}$.

As said, the goal is to carry all $t$ persons, initially in $c_{I}$, to $c_{G}$ using $p$ planes, minimizing both the makespan and the cost of the plan. In order to ease the identification of the true Pareto Front, a symmetry constraint is added: $\forall i \in$ $[1, n], d_{i}^{I}=d_{i}^{G}$ and from thereon we will refer to a unique vector $d$.

Without loss of generality, all pairs $\left(d_{i}, c_{i}\right)$ are assumed to be pairwise distinct. Otherwise, the 2 cities can be "merged" and the resulting $n-1$ cities problem is equivalent to the original $n$ cities problem, as there exist no city capacity constraints. Finally, we only consider cases where $t \geq p$, as the problem is otherwise trivial.

\subsection{Pareto Optimal Plans}

Let us make another simplifying assumption:

Assumption A1: $\forall(i, j) \in[1, n]^{2}, d_{i}+d_{j}<d_{i j}{ }^{3}$. Then the following holds.

Proposition: Pareto-optimal plans are plans where exactly $2 t-p$ (possibly identical) central cities are used by a flight.

Proof: Consider a plan where a person flies from $C_{i}$ to $C_{j}$. Using the same plane, the same person could fly instead from $C_{i}$ to $C_{G}$, and the plane would return empty to $C_{j}$. The plan could continue unchanged from thereon: because

\footnotetext{
3 This might look unrealistic in real-world logistic domain. However, we hypothesize that the proposition still holds with the weaker condition that for any cities $C_{i}, C_{j}, C_{k}$ (if we state the cost of $C_{I}$ and $C_{G}$ are respectively $C_{0}$ and $C_{n+1}$ ), $d_{i k} \leq d_{i j}+d_{j k}$ (triangle inequality).
} 
of the hypothesis on makespans, the needed resource would be in $C_{j}$ on time. Moreover, the total cost is unchanged, and the total makespan is lower or equal to the original one: the new plan thus Pareto-dominates the original one.

Iterating the same reasoning for each person, and each empty plane, we conclude that there are no flights between central cities in Pareto-optimal plans. Thus bringing the $t$ persons from $C_{I}$ to $C_{G}$ will amount to carry each person through one central city: $t$ flights will be needed from $C_{I}$ to one $C_{i}$, then $t$ flights from $C_{i}$ to $C_{G}$. Finally, because planes do not need to come back from $C_{G}$ in the end, only $t-p$ flights back empty will be needed, possibly through some different central cities - hence the result.

PPPs and Admissible PPPs: According to the above proposition, a Possibly Pareto-optimal Plan (PPP) is defined by 2 tuples, namely $e \in[0, n]^{t}$ for cities involved in eastbound flights, and $w \in[0, n]^{t-p}$ for westbound flights. Nevertheless, $e$ and $w$ do not hold any information about which plane will land in a particular city. This is the reason why there exists many feasible schedules, i.e., schedules that actually are feasible plans for $p$ planes ${ }^{4}$ using the corresponding $4 t-2 p$ edges. There are at most $n^{(2 t-p)}$ possible PPP but it is clear that the set of PPPs contains many redundancies, that can easily be removed by ordering the indices:

Definition: An admissible PPP is a pair of $E \times W$, where $E=\left\{e \in[1, n]^{t} ; \forall i \in\right.$ $\left.[1, t-1], d_{e_{i}} \geq d_{e_{i+1}}\right\}$ and $W=\left\{w \in[1, n]^{t-p} ; \forall i \in[1, t-p-1], d_{w_{i}} \geq d_{w_{i+1}}\right\}$.

Number of admissible PPPs: Let $K_{k}^{m}$ be the set of $k$-multicombinations (or multi-subset of size $k$ ) with elements in a set of size $m$. The cardinality of $K_{k}^{m}$ is $\Gamma_{k}^{m}=\left(\begin{array}{c}m+k-1 \\ k\end{array}\right)$. As $E$ is in bijection with $K_{t}^{n}$, and $W$ with $K_{t-p}^{n}$, the number of PPP is $\Gamma_{t}^{n} \Gamma_{t-p}^{n}$, i.e., $\left(\begin{array}{c}n+t-1 \\ t\end{array}\right)\left(\begin{array}{c}n+(t-p)-1 \\ t-p\end{array}\right)$.

Cost of a PPP: Given the PPP $C=(e, w) \in E \times W$, the cost of any plan using only the cities in $e$ and $w$ is uniquely defined by $\operatorname{Cost}(C)=\sum_{e_{i} \in e} c_{e_{i}}+\sum_{w_{i} \in w} c_{w_{i}}$.

Makespan of a PPP: The makespan of a PPP is thus that of the shortest schedule that uses its $4 t-2 p$ edges in a feasible way. Trivial upper and lower bounds for the shortest makespan of a PPP $C$ are respectively $M_{S}(C)$, the makespan of the sequential plan (i.e., that of the plan for a single plane that would carry all persons one by one), and $M_{L}(C)$, the makespan of the perfect plan where none of the $p$ planes would ever stay idle. As discussed in Section 3, these bounds are useful to prune the set of PPPs.

$$
M_{S}(C)=2\left(\sum_{e_{i} \in e} d_{e_{i}}+\sum_{w_{i} \in w} d_{w_{i}}\right) \quad \quad M_{L}(C)=\frac{M_{S}(C)}{p}
$$

\footnotetext{
${ }^{4}$ Most of them are probably not Pareto-optimal, but w.r.t the previous proposition, any schedule resulting from a larger tuple $e$ or $w$ would be Pareto-dominated.
} 
Greedy domination: Given two PPP $C$ and $C^{\prime}, C$ greedily dominates $C^{\prime}$ if $M_{S}(C) \leq M_{L}\left(C^{\prime}\right)$ and $\operatorname{Cost}(C) \leq \operatorname{Cost}\left(C^{\prime}\right)$.

\subsection{Computing the Shortest Makespan}

Flight patterns Clearly, within a PPP, all possible plane moves can be categorized into only 3 patterns:

P1: plane leaves $C_{I}$ (non empty), flies eastward to city $C_{i}$, and goes on to $C_{G}$.

P2: plane leaves $C_{G}$ (empty), flies westward to city $C_{i}$, and goes on to $C_{G}$.

P3: two planes are involved here; first plane leaves $C_{I}$ (with a passenger), flies to city $C_{i}$, and goes back empty to $C_{I}$; second plane leaves $C_{G}$ empty, flies to $C_{i}$, and flies back with the passenger to $C_{G}$. Note that there can be some delay between the drop-off of the person at the central city, and the arrival of the second plane.

Given a feasible plan using only the three above patterns, let $\alpha_{E}, \alpha_{W}$, and $\beta$ be the numbers of effective $\mathrm{P} 1, \mathrm{P} 2$, and $\mathrm{P} 3$ patterns respectively. It is clear that $\beta$ entirely determines $\alpha_{E}$ and $\alpha_{W}$, as $\alpha_{W}=t-p-\beta$ and $\alpha_{E}=t-\beta$. Considering a PPP C, it is possible for a given $\beta$ to have multiple choices for the cities involved in P3. Each choice is denoted $\beta_{\text {set }}$ and the set of $\beta_{\text {set }}$ the $\beta$-PowerSet.

The optimal makespan for a given admissible PPP $C$ is the lowest makespan obtained for all $\beta_{\text {set }} \in \beta$-PowerSet. Once the optimal makespan for a couple $\left(C, \beta_{\text {set }}\right)$ determined, iterating over the $\beta$-PowerSet held by $C$ returns the optimal makespan for $C$. Finally, iterating the process over the set of PPP returns the Pareto Front for the considered instance.

The method to compute the optimal makespan for a particular couple $\left(C, \beta_{\text {set }}\right)$ is broken down into two steps. In a first step, each $\beta_{\text {set }}$ defines a subproblem without any P3 that is easy to solve. The second step is to take into account the P3 patterns in $C$. After detailing these two steps, we will give a constructive proof that the obtained makespan is optimal.

Step 1: Handling P3-free PPPs For a given $\left((e, w), \beta_{\text {set }}\right)$ denote $e^{\prime}=e \backslash \beta_{\text {set }}$, i.e. the tuple $e$ from which all elements of $\beta_{\text {set }}$ have been removed, and $w^{\prime}=$ $w \backslash \beta_{\text {set }}$ defined similarly. As a result, $\left(\left(e^{\prime}, w^{\prime}\right), \emptyset\right)$ is the subproblem of $\left((e, w), \beta_{\text {set }}\right)$ that does not contain any P3 $\left(\beta^{\prime}=0\right)$.

For a PPP with $\beta=0$, greedy Algorithm 1 dispatches the longest flight durations first, assigning them to the available planes with shortest 'private' makespan (who have yet flown the less), ending with the one-way last flights from $C_{I}$ to $C_{G}$ (planes end in $C_{G}$ ). The algorithm returns the flight durations $\left(D_{k}^{1}\right)$ for all planes $k$ (to be used in the second step), and the optimal makespan for the subproblem is obviously $\max _{k}\left(D_{k}^{1}\right)$.

Step 2: Tackling Patterns P3 The second step consists in dispatching the durations of P3 patterns among the planes according to their previous flight durations $\left(D_{k}^{1}\right)_{k=1, \ldots, p}$, by sequentially assigning the longest P3 flight to the two 


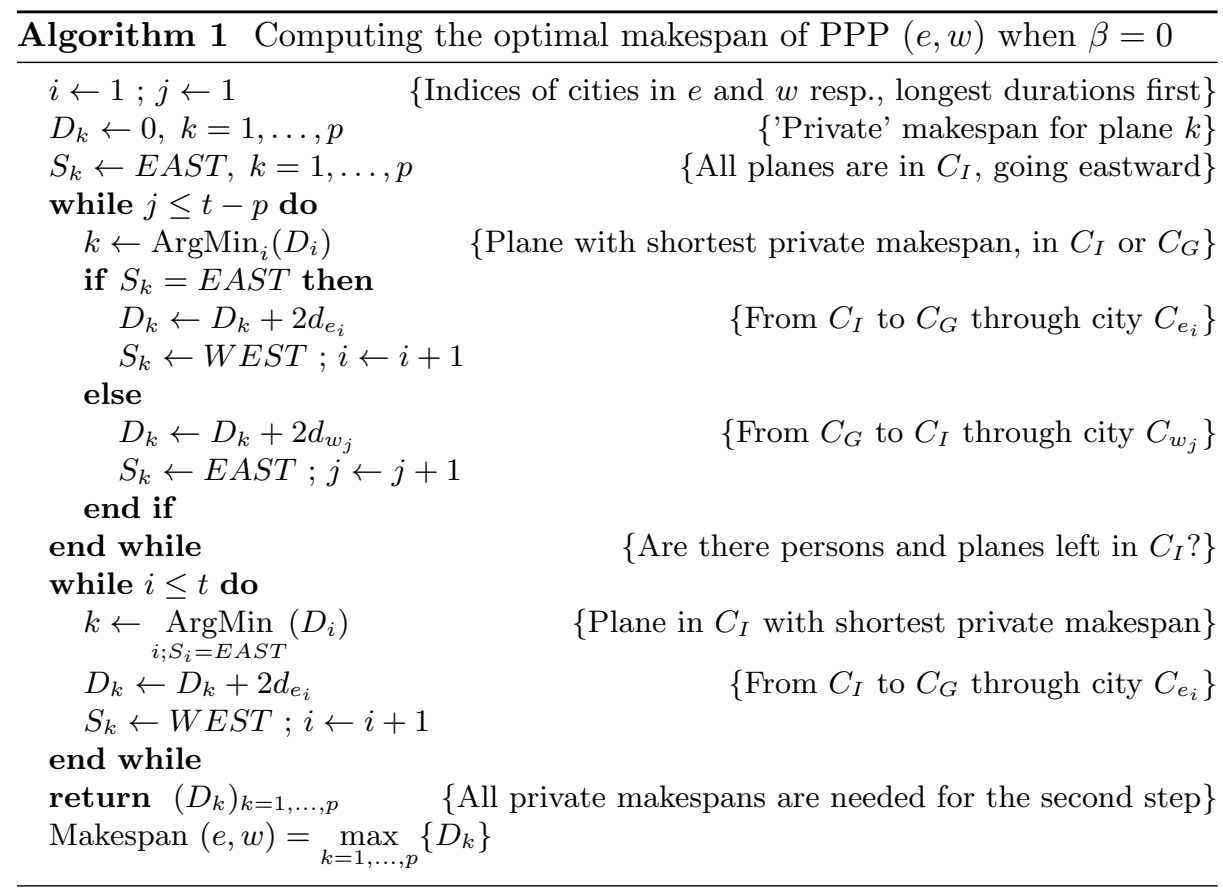

planes with the smallest current flight durations. This can be performed greedily again, with a slightly modified version of the Algorithm 1, if we only consider the flight durations.

However, within a P3 pattern, if the plane coming from $C_{G}$ lands in the central city before the person has yet arrived from $C_{I}$, it has to wait. Consequently, it is possible that the makespan of the plan is not simply the sum of the pattern durations. Indeed, the described algorithm is not taking into account the possibility of a waiting point and this is the reason why we first have to discuss the construction of a feasible plan according to the final vector of durations $\left(D_{k}^{2}\right)_{k=1, \ldots, p}$ before discussing the optimality of $\max _{k=1, \ldots, p}\left\{D_{k}^{2}\right\}$ as the makespan of the associated PPP.

Proposition: It is always possible to construct a feasible plan with the makespan returned by the two-steps method described above.

Proof by construction: Considering a P3 pattern performed by planes $p_{1}$ in $C_{G}$ and $p_{2}$ in $C_{I}$ through city $C_{i}$. Their schedules will look something like:

$$
\begin{aligned}
& p_{1}: C_{I} \rightarrow \ldots \rightarrow C_{G} \rightarrow \grave{C}_{i} \rightarrow C_{G} \rightarrow \ldots \rightarrow C_{G} \\
& p_{2}: C_{I} \rightarrow \ldots \rightarrow C_{I} \rightarrow \stackrel{\ominus}{C}_{i} \rightarrow C_{I} \rightarrow \ldots \rightarrow C_{G}
\end{aligned}
$$

Let $\checkmark$ denote the time $t_{1}$ when plane $p_{1}$ arrives in $C_{i}$ and $\diamond$ the time $t_{2}$ when plane $p_{2}$ (with the person) arrives in $C_{i}$. If $t_{2}>t_{1}$ then plane $p_{1}$ will have to wait 
$t_{2}-t_{1}$ in $C_{i}$ before flying back to $C_{G}$ with the person. But the duration vector $\left(D_{k}^{2}\right)$ returned by the two steps algorithm is computed assuming no waiting point. Consequently, the proposition is equivalent to assert that we can always build a plan without any waiting point.

In order to do so, for each P3, the idea is to perform the westward part as early as possible, and on the opposite, to perform the eastward part as late as possible, thus ensuring that there is no waiting time.

In order to construct such an optimal plan, we will remember the cities of every plane and every pattern during both previous steps of the algorithm. From there on, let us consider now only planes that have to perform at least one P3 pattern.

1. For each plane, select the one with the maximum number of P3 patterns to be performed. In case of tie, select the plane with longest P3 duration, or the plane with the largest current 'private' makespan.

2. Construct a partial schedule with only P1 and P2 patterns (Step 1 above).

3. For every 'not already started' P3 pattern, add its eastward part at the end of the schedule by descending order of durations.

4. For every 'already started' P3 pattern, add its westward part at the beginning of the schedule by ascending order of durations.

Example: Considering $t=7, p=3, d=(2,4,6), C=(3,3,2,2,2,1,1)(3,3,2,1)$ and $\beta_{\text {set }}=\{3,2,1\}$ leads to the sub-problem $C^{\prime}=(3,2,2,1)(3)$ with $t^{\prime}=4$. Step 1 above gives the 'private' makespans $D_{k}^{1}$ in the table below. Adding the P3 patterns (Step 2) gives the 'private' makespans $D_{k}^{2}$. The complete schedule can then be built according to the method described above.

$$
\begin{array}{|c|c|c|c|l|l}
\hline p_{i} & D_{i}^{1} & D_{i}^{2} & \mathrm{P} 3 \\
\hline p_{1} & 12 & 32 & 2 & p_{1}: c_{I} \rightarrow c_{2} \rightarrow c_{G} \rightarrow c_{3} \rightarrow c_{G} \rightarrow c_{2} \rightarrow c_{G} \rightarrow c_{1} \rightarrow c_{G} \\
p_{2} & 24 & 28 & 1 & p_{2}: c_{I} \rightarrow \iota_{1} \rightarrow c_{I} \rightarrow c_{2} \rightarrow c_{G} \rightarrow c_{3} \rightarrow c_{I} \rightarrow c_{2} \rightarrow c_{G} \\
p_{3} & 8 & 32 & 3 & p_{3}: c_{I} \rightarrow \diamond_{3} \rightarrow c_{I} \rightarrow \iota_{2} \rightarrow c_{I} \rightarrow c_{3} \rightarrow c_{G}
\end{array}
$$

Hence, there is no waiting time within P3s, and the optimal makespan is 32 .

Proposition: For a given PPP $C$ and $\beta_{\text {set }}$, the algorithm returns the optimal makespan.

Proof: The incompressible time to transport all passengers, according to a given $\beta_{\text {set }}$ is $T=4 \sum_{i \in \beta_{\text {set }}} d_{i}+2 \sum_{i \in\left\{e^{\prime}, w^{\prime}\right\}} d_{i}$. A theoretical optimal plan with this pattern repartition is a plan without any waiting point for any plane. The above algorithm gives the optimal distribution of the set of times into $p$. Then, if a plan can be constructed with such a makespan, it is optimal for the PPP and the repartition of patterns. As it exists a method to construct such a plan, we can conclude that the algorithm is optimal for the PPP C and $\beta_{\text {set }}$. 
Complexity: Given a PPP, the worst case occurs when $w \subset e$ and $w_{i} \neq w_{j}$ if $i \neq j$. Hence, for each value of $\beta$ there are $\left(\begin{array}{c}t-p \\ \beta\end{array}\right)$ possible $\beta_{\text {set }}$. As $0 \leq \beta \leq t-p$, we will perform $2^{t-p}$ iterations of the two step algorithm. A large upper-bound for the whole PPP set is hence $2^{t-p}\left(\begin{array}{c}n+t-1 \\ t\end{array}\right)\left(\begin{array}{c}n+(t-p)-1 \\ t-p\end{array}\right)$.

However, if an upper bound on $\beta$ is given by $t-p$, a tighter upper-bound can be found as explained by the following example and due to the fact that the worst case situation for a PPP rarely occurs in the whole PPP set, the real number of iterations for a given instance is far from the above bound.

Example: Considering $C=(3,1,1)(2,1)$, the trivial upper bound is equal to two but actually, it is impossible to operate a P3 using the city $C_{2}$ since it is not in the tuple $e$.

\section{ZenoSolver}

ZenoSolver is a C++11 software dedicated to generate and exactly solve MuLTiZenoTravel instances. Firstly, it allows to tune every parameter in order to adjust the difficulty or to obtain different shapes of Pareto Fronts. In particular, vectors $c$ and $d$ are generated using two user-defined functions, $f$ and $g$, such that $c_{i}=x_{c} f(i)+y_{c}$ and $d_{i}=x_{d} g(n-i)+y_{d}$, insuring that both objectives are conflicting. Second, ZENoSolver outputs the corresponding PDDL file ${ }^{5}$, that can be directly used by any standard AI planner.

Finally, ZenoSolver computes the true Pareto Front using the algorithm described in Section 2, iterating over $E \times W$, storing for each value of the total cost the PPP with best makespan to date, without explicitly constructing the set of admissible PPPs. Using the Greedy domination, ZENoSolver implements a pruning method that checks if the current PPP is dominated by any other PPP already stored. As noted, the optimal makespan is lower or equal than the upper bound $M_{S}$, leading to an efficient pruning. Indeed, as PPPs are generated in an approximated increasing order [4], this avoids to iterate over the whole set to check the domination criterion.

Determining if the current PPP is dominated has complexity $O(h)$ where $h$ is the number of different total costs. An obvious upper-bound for $h$ is given by $(2 t-p)\left(\max _{i}\left(c_{i}\right)-\min _{i}\left(c_{i}\right)\right)$. However, in practice, $S$ seems to have the same order of magnitude than the exact Pareto Front. In addition, $S$ is the only structure kept in memory, thus, from this point of view, ZENOSOLVER turns out to be near-optimal regarding the memory usage (see Table 1 ).

\subsection{Empirical Performances}

Empirical complexity The number of iterations is influenced by the number of PPPs but also by their structure. Indeed, increasing $n$ does not significantly

\footnotetext{
${ }^{5}$ Planning Domain Definition Language, universally used now in AI Planning to describe domains and instances.
} 

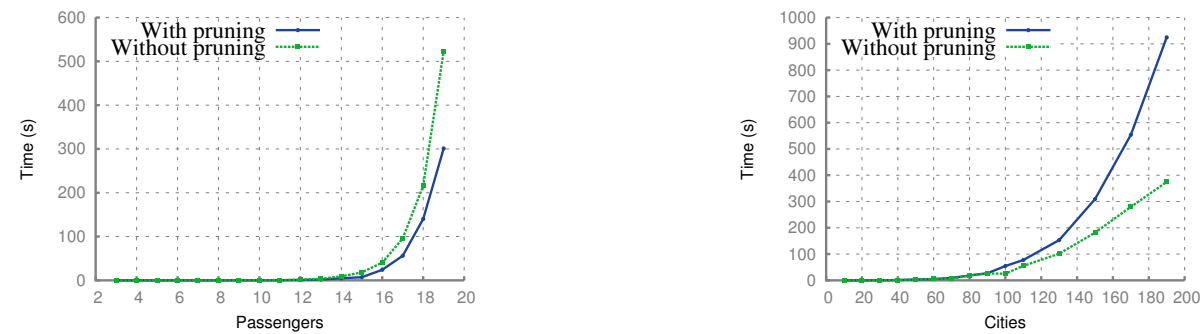

Fig. 2: Time function of $t$ (left) or $n$ (right) for $f(i)=g(i)=i$.
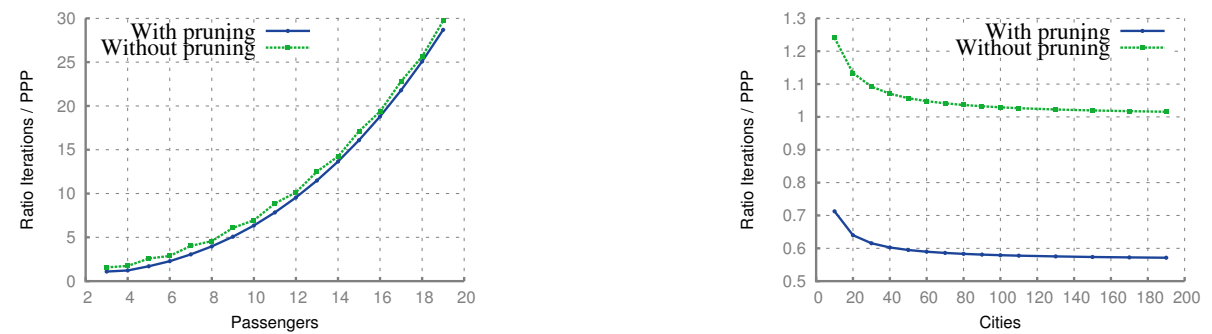

Fig. 3: Ratio of iterations over the number of PPPs, function of $t$ (left) or $n$ (right) for $f(i)=g(i)=i$.

impact the average number of iterations per PPP since the upper-bound is $2^{t-p}$. On the opposite, increasing $t$ leads to a dramatic growth of both the upperbound and the average number of iterations per PPP. Figure 2, which displays the time vs $t$ or $n$ plots, confirms this remark: it requires the same CPU time for $t=18$ than for $n=165$.

Pruning or not pruning ? The benefits of the pruning method strongly rely on the average number of iterations per PPP: Pruning becomes more efficient as $t$ increases, as shown by Figure 2. Furthermore, increasing $n$ while pruning can degrade performances, even if there are less iterations than PPPs (Figure 3 compared to Figure 2). Note that the number of iterations follows the number of PPPs while increasing $n$, but explodes with $t$, which is in line with the previous remark.

Also, the efficiency pruning seems to be instance-dependent. Fixing $n, t$ and $p$, different generating functions result in different numbers of iterations and CPU times, as demonstrated by Figure 4 . There are however some clear cases in favor of pruning, e.g. with $n=t=9$ : ZENOSOLVER requires $1.26 \times 10^{9}$ iterations and 2222 seconds without pruning. Using pruning, for $f(i)=\sqrt{i}$ and $g(i)=i$, it requires only 119000 iterations performed in 26 seconds, but 36000 iterations in 53 seconds with $f(i)=\log (i)$ and $g(i)=\sqrt{i}$. In general, using concave generating functions leads to more optimistic conclusions regarding the benefits of pruning PPPs. 

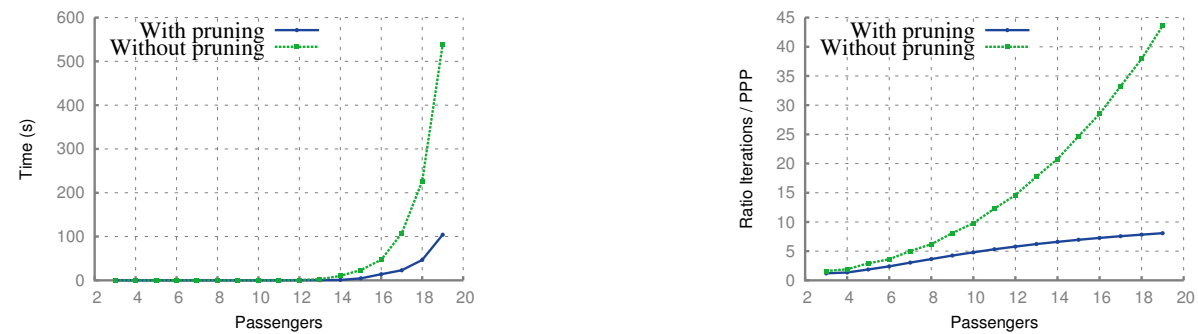

Fig. 4: Time and ratio for generating functions $f(i)=g(i)=\log (i)$.

Table 1: Increasing simultaneously $n$ and $t$ with $f(i)=g(i)=i$.

\begin{tabular}{|c|c|c|c|c|c|c|c|}
\hline $\mathrm{n}$ & $\mathrm{t}$ & $\mathrm{p}$ & PPP Size & Iterations & S Size & Front Size & Time $(\mathrm{ms})$ \\
\hline 3 & 3 & 2 & 30 & 33 & 9 & 5 & 0 \\
4 & 4 & 2 & 350 & 408 & 19 & 10 & 1 \\
5 & 5 & 2 & 4410 & 6387 & 33 & 17 & 6 \\
6 & 6 & 2 & 58212 & 109831 & 51 & 26 & 117 \\
7 & 7 & 2 & 792792 & 1930385 & 73 & 37 & 2278 \\
8 & 8 & 2 & 11042460 & 34648348 & 99 & 50 & 43572 \\
9 & 9 & 2 & 156434850 & 630225670 & 129 & 65 & 1036772 \\
10 & 10 & 2 & 2245709180 & 11600589455 & 163 & 82 & 20785211 \\
\hline
\end{tabular}

\subsection{Reference Large Instances}

As mentioned in the introduction, the combinatorial multi-objective optimization domain suffers from a lack of benchmarks with a known Pareto Front but also with a concave or non-regular shapes ${ }^{6}$.

Even if anyone can generate different instances by tuning ZENoSolver parameters to obtain the desired front shape with accuracy, we identified some large instances with totally different front shapes and complexities as displayed in Figure 5: They could be a basic set of representative instances for MultiZenoTRAVEL, allowing fair comparisons between various solvers and approaches. Note that more large instances with different complexities can be found on the website of the Descarwin Project https://descarwin.lri.fr.

Table 2 gives the parameters used by ZENOSOLver to build them, as well as some statistics about their complexity. The choice of the generating functions is purely empirical, guided by the fact that we would like to obtain mainly piecewise concave fronts with uneven point distributions. This is why none of these fronts is linear, though some seem to be at large scale (see detailed insets in some plots). Also note the non-uniform distribution of the points on the Instances 3, and the few Pareto points of the Instance 4 in spite of the complexity of this instance (26 persons), due to the small ratio $\frac{p}{t}$. The generating time strongly varies from some

\footnotetext{
${ }^{6}$ In the context of discrete optimization, the word "concave" seems rather abusive. However, we will call here concave parts of a Pareto front where all points are above the segment made of the two extreme points, w.r.t. the direction of optimization.
} 

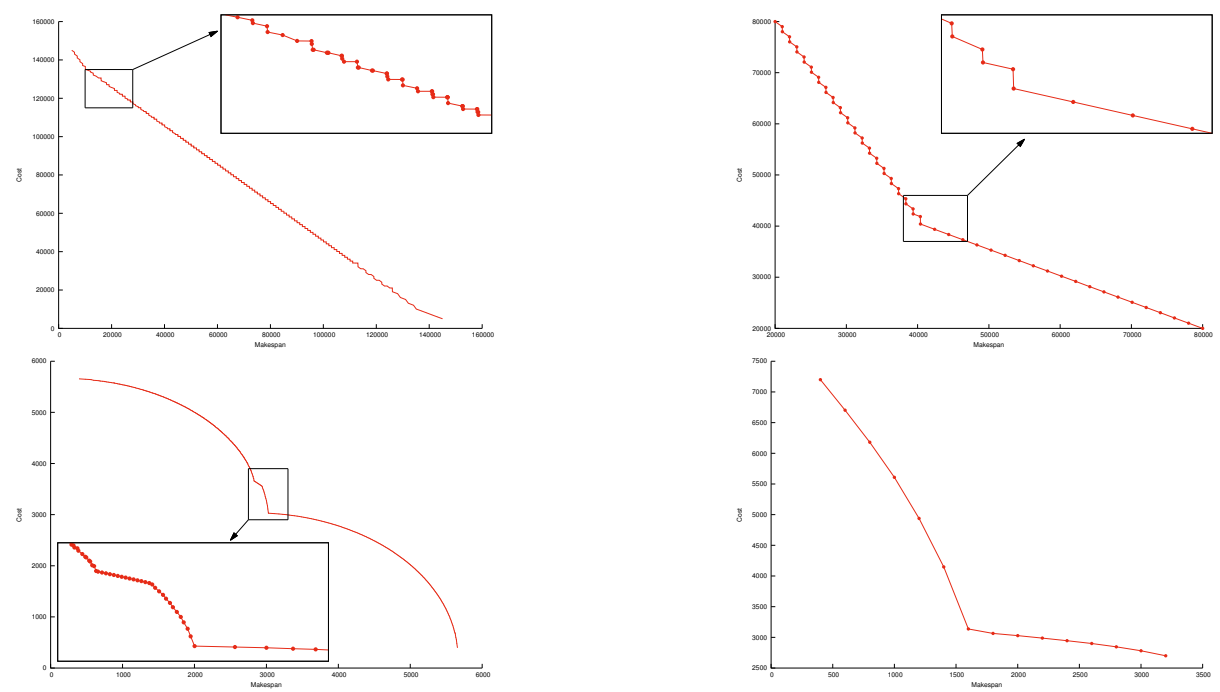

Fig. 5: True Pareto Fronts for the instances described by Table 2. Remember that these Pareto fronts are made of discrete points: the lines are visual helps to make the general shape clear.

Table 2: Large instances: parameters and generation statistics.

\begin{tabular}{|c|c|c|c|c|c|c|c|c|c|c|}
\hline Inst. & $n$ & $t$ & $p$ & \multicolumn{2}{|c|}{ Generating functions } & Pareto\# & $h$ & $\mathrm{PPP}(\mathrm{k})$ & Iter. $(\mathrm{k})$ & Time \\
\hline 1 & 20 & 6 & 2 & 5. $\quad(i \bmod 2)$ & $(i \bmod )$ & 409 & 4015 & 1568220 & 3317140 & $16 \mathrm{~h} 46$ \\
\hline 2 & 3 & 21 & 2 & $\frac{-}{2} \imath+$ & 10 & 61 & 861 & 53 & 233 & $2006 \mathrm{~s}$ \\
\hline 3 & 200 & 3 & 2 & $\sqrt{i}$ & $\sqrt{i}$ & 538 & 4963 & 270680 & 3906 & $1845 \mathrm{~s}$ \\
\hline 4 & 8 & 26 & 25 & $\sqrt{i}$ & $i$ & 15 & 190 & 34176 & 60457 & $4240 \mathrm{~s}$ \\
\hline
\end{tabular}

minutes up to hours and thus confirm dependency on the generating functions of the ZENoSOLVER complexity.

\section{Conclusion and perspectives}

This paper has extended the MultiZenoTravel test suite in multi-objective AI planning. Furthermore, not only did we provide here a general approach to generate more complex Pareto fronts than in our previous work [2], but we also proposed here ZENOSOLVER, an exact solver that is provably able to exactly solve the multi-objective optimization problem (i.e., to identify the true Pareto front) for even very large instances. The complete code is publicly available at https://descarwin.lri.fr, making it easy for everyone to generate his/her own benchmark instances. However, we also provided in this paper a few typical instances that exhibit very different shapes of Pareto Fronts, for different levels of complexity. 
The proposed benchmark suite opens the floor to sound comparative experiments in a combinatorial domain where, as far as we know, no ground truth (i.e., true Pareto front) existed for large instances. On-going work is concerned with using these benchmarks to compare different multi-objective optimization algorithms. Preliminary results [7] have already confirmed that Pareto-based Evolutionary Algorithms outperform the basic weighted sum aggregation in the case of complex non-convex Pareto fronts. However, deeper experiments should be made with state-of-the-art decomposition algorithms in which the different components of the decomposition cooperate (e.g., from the MOEA/D family [6]). In particular, in the AI planning domain, using these benchmarks will hopefully lead to more sound comparisons between Pareto and non-Pareto planners (see e.g., $[5,3])$.

\section{References}

1. C. Igel, N. Hansen, and S. Roth. Covariance matrix adaptation for multi-objective optimization. Evolutionary Computation, 15(1):1-28, 2007.

2. M. R. Khouadjia, M. Schoenauer, V. Vidal, J. Dréo, and P. Savéant. Multi-Objective AI Planning: Evaluating DAE-YAHSP on a Tunable Benchmark. In R.C. Purshouse et al., editor, "th EMO, pages 36-50. LNCS 7811, Springer Verlag, 2013.

3. M. R. Khouadjia, M. Schoenauer, V. Vidal, J. Dréo, and P. Savéant. Pareto-Based Multiobjective AI Planning. In Francesca Rossi, editor, IJCAI, pages 2321-2328. AAAI Press, 2013.

4. D. E. Knuth. The Art of Computer Programming, Generating All Tuples and Permutations, volume 4. 2005.

5. Sroka M. and Long D. Exploring Metric Sensitivity of Planners for Generation of Pareto Frontiers. In K. Kersting and M. Toussaint, editors, $6^{\text {th }}$ STAIRS, pages 306-317. IOS Press, 2012.

6. Zhang Q. and Li H. A multi-objective evolutionary algorithm based on decomposition. IEEE Transactions on Evolutionary Computation, 11(6):712-731, 2007.

7. Alexandre Quemy, Marc Schoenauer, Vincent Vidal, Johann Dréo, and Pierre Savéant. Solving large multizenotravel benchmarks with divide-and-evolve. In C. Daenens et al., editor, Proc. LION'9. Springer Verlag, 2015. To appear.

8. M. Schoenauer, P. Savéant, and V. Vidal. Divide-and-Evolve: a New Memetic Scheme for Domain-Independent Temporal Planning. In J. Gottlieb and G. Raidl, editors, $6^{\text {th }}$ EvoCOP, pages 247-260. LNCS 3906, Springer Verlag, 2006.

9. E. Zitzler, K. Deb, and L. Thiele. Comparison of multiobjective evolutionary algorithms: Empirical results. Evolutionary Computation, 8(2):173-195, 2000. 